\title{
Effects of leukocyte- and platelet-rich plasma on tendon disorders based on in vitro and in vivo studies (Review)
}

\author{
XUELI LIU ${ }^{1,2^{*}}$, RONG ZHANG ${ }^{2 *}$, BIN ZHU ${ }^{1}$, YUJIE LI $^{1}$, XINYUE LIU $^{1}$, SHENG GUO $^{3}$, \\ CHENGLONG WANG ${ }^{3}$, DINGXUAN WANG ${ }^{1}$ and SEN LI ${ }^{1,3}$ \\ ${ }^{1}$ Department of Physical Education, Southwest Medical University, Luzhou, Sichuan 646000; ${ }^{2}$ Department of Rehabilitation, \\ Sichuan Vocational College of Health and Rehabilitation, Zigong, Sichuan 643000; ${ }^{3}$ Spinal Surgery Department, \\ Hospital (Traditional Chinese Medicine) Affiliated to Southwest Medical University, Luzhou, Sichuan 646000, P.R. China
}

Received November 3, 2020; Accepted March 22, 2021

DOI: $10.3892 / \mathrm{etm} .2021 .10071$

\begin{abstract}
Tendon-related disorders are common musculoskeletal system disorders in clinical practice, accounting for $30-50 \%$ of all sports-related injuries, and they are difficult to treat due to the hypovascular structure of the tendons. Platelet-rich plasma (PRP), including pure PRP and leukocyte- and platelet-rich plasma (L-PRP), has been attracting increasing attention, as it may stimulate tissue regeneration through the release of growth factors and cytokines. The aim of the present review was to provide a summary of the effects of L-PRP on tendon disorders and the underlying mechanisms through a comprehensive examination of the published literature, including in vitro, animal and clinical studies. It has been demonstrated that L-PRP results in comparatively greater pain relief and improved function in patients suffering from tendon disorders. Furthermore, L-PRP may exert its effects through a diverse range of mechanisms, such as neovascularization, cell proliferation and differentiation of tendon/progenitor stem cells into tenocytes, as well as extracellular matrix reorganization by transforming type III to type I collagen fibers. It has also been indicated that the effects of leukocytes in L-PRP depend on the biological state of the injured tissue and its surrounding microenvironment. L-PRP is beneficial and promotes tendon
\end{abstract}

Correspondence to: Dr Sen Li, Spinal Surgery Department, Hospital (Traditional Chinese Medicine) Affiliated to Southwest Medical University, 182 Chunhui Road, Longmatan, Luzhou, Sichuan 646000, P.R. China

E-mail: jht187@163.com

Professor Dingxuan Wang, Department of Physical Education, Southwest Medical University, 1 Xianglin Road, Longmatan, Luzhou, Sichuan 646000, P.R. China

E-mail: 635739608@qq.com

*Contributed equally

Key words: leukocyte- and platelet-rich plasma, platelet-rich plasma, tendon healing, leukocyte, tendon disorders, inflammation, tendinopathy healing at the early stage, whereas it is likely detrimental to the repair of tendon at a later stage because of the risk of excessive catabolic and inflammatory responses. Overall, the application of L-PRP in tendon disorders appears to be a promising field that is worthy of further research.

\section{Contents}

1. Introduction

2. Effects of L-PRP on tendon healing based on basic studies

3. Effects of L-PRP on tendon healing based on clinical studies

4. Conclusion and perspectives

\section{Introduction}

Tendons are the uniaxial connective tissue component of the musculoskeletal system; they are responsible for transmitting the contractile force between musculoskeletal tissues and they are viscoelastic and sensitive to different degrees of strain (1). When a tendon is acutely injured or subjected to repeated excessive use (collectively referred to as tendon disorders), its structure and function may be altered, affecting the ability of millions of individuals to perform physical exercise or work. Regrettably, tendon disorders are common and the most frequent injuries include the flexor and extensor tendons of the hand (incidence rates of 4.83 and 17.87/100,000 individuals per year, respectively), the Achilles tendon (11.33/100,000 per year) and the rotator cuff tendon (3.73/100,000 per year) (2).

The treatment options for tendon disorder mainly include surgical repair, mechanical stimulation (sports rehabilitation) and topical anti-inflammatory drugs, supplemented by interventions such as physical therapy and tissue engineering. Recent studies have indicated that platelet-rich plasma (PRP) is a promising therapeutic approach for musculoskeletal injuries, as it is simple, safe, cost-effective and minimally invasive (3-5). PRP has been indicated to be an optimal autologous biological blood-derived product that releases high concentrations of growth factors and cytokines on injection, such as platelet-derived growth factor (PDGF), vascular endothelial 
growth factor (VEGF), basic fibroblast growth factor (bFGF), transforming growth factor- $\beta$ (TGF- $\beta$ ) and insulin-like growth factor-1 (IGF-1), which contribute to tendon healing. The functions of growth factors are summarized in Table I (6-9). These growth factors perform their respective functions, where they could promote tendon healing by promoting angiogenesis, cellular proliferation, cellular differentiation and collagen production. Previous research has indicated that PRP, including leukocyte- and PRP (L-PRP) and pure PRP (P-PRP) (10), has a key function in promoting tendon regeneration, repair and healing, as it may enhance the formation and healing of the tendon tissue structure.

Although several studies have reported favorable clinical outcomes after the application of PRP, other studies have indicated no significant improvement in pain or tendon functions after L-PRP treatment (11-13). These conflicting data may be mainly attributed to variations in PRP preparation procedures, disease stage and patient-related factors (12). A previous study investigated the cellular components of PRP and indicated that the leukocyte concentration varies significantly among different PRP preparations as compared with platelets and fibrinogen (14). Furthermore, the leukocyte concentration and composition strongly affect the quality of the PRP. Most previous studies did not specify the type of PRP in terms of whether it did or did not contain leukocytes $(15,16)$. However, due to the controversy regarding the efficacy of PRP, an increasing number of studies have clarified the specific components of PRP (17).

The present review provided a summary of the effects of L-PRP on tendon disorders and the underlying mechanisms through a comprehensive examination of the relevant published literature, including clinical, animal and in vitro studies, with the aim of providing evidence supporting the application of L-PRP in tendon disorders in the future and resolving the controversy surrounding the use of PRP to a certain extent.

\section{Effects of L-PRP on tendon healing based on basic studies}

General. Tendon healing is a complex process, which largely occurs through three overlapping phases: Inflammation, proliferation and remodeling $(1,3,18)$. L-PRP accelerates the process of tendon healing, with the platelets initiating wound repair by releasing locally acting growth factors via degranulation of $\alpha$-granules (6). The growth factors released from L-PRP and their effects on the molecular structure of the tendon are presented in Fig. 1. L-PRP can promote angiogenesis, proliferation and differentiation of TSCs into tenocytes, in addition to type I collagen production.

Angiogenesis. A number of in vitro and animal studies have reported that administration of L-PRP significantly increased angiogenesis during the early phase of the tendon repair process (19-24). It has been suggested that poor vascularity is a major factor limiting tendon healing capacity (25).

L-PRP contains higher concentrations of growth factors involved in neovascularization compared with whole blood or platelet-poor plasma, such as VEGF $(6,20,26-29)$ and PDGF $(6,20,28,29)$, which together promote vessel wall permeability, as well as the growth and proliferation of vascular endothelial cells. Increased growth factor concentration and expression may be an intrinsic mechanism involved in inducing angiogenesis as part of the tissue repair process. Following angiogenesis, the resumption of blood flow promotes the recruitment of reparative cells from the peripheral blood and bone marrow, so that the intrinsic tenocytes and fibroblasts of surrounding tissues migrate to the injured site and begin to proliferate and synthesize collagen (30).

Therefore, one of the mechanisms through which L-PRP accelerates tendon healing is the enhancement of blood supply to the injured soft tissue. The neovascularization proceeds along the surface of the epitenon, passing through normal vascular areas and providing the injured area with extrinsic cells, nutrients and growth factors (7). This effect is mediated by angiogenic growth factors, such as VEGF and PDGF, which are contained in L-PRP. Furthermore, Kobayashi et al (31) reported that the leukocyte concentration in L-PRP was positively correlated with the concentrations of PDGF and VEGF, while it was negatively correlated with the concentration of bFGF (32). In conclusion, L-PRP accelerates the angiogenic process and, subsequently, leads to the acceleration of the tendon healing process.

Cell proliferation and differentiation. There are two cell types in tendons: Tenocytes, which are the predominant cell type, and the tendon stem/progenitor cells (TSCs), which represent a small proportion $(<5 \%)$ of the total tendon cells. Although certain cell culture studies have indicated that PRP treatment increases tenocyte proliferation $(26,27)$, the rate of tenocyte proliferation is limited after tendon injury. On the contrary, TSCs have a high proliferation rate, which may be further enhanced by PRP $(33,34)$. Also, similar to other adult stem cells, TSCs are able to self-renew and differentiate into tenocytes that are responsible for the maintenance and repair of the tendons $(34,35)$.

Regarding the effect of L-PRP on tendon cell proliferation and differentiation, studies have indicated that it may be attributed to the high amount of growth factors in L-PRP, including PDGF $(6,20,28,29)$, TGF- $\beta(6,20,31,36)$ and IGF-1 (32). Those previous in vitro or animal studies demonstrated that these growth factors are present at high concentrations in L-PRP and have an important role in cell proliferation and tenocyte differentiation of TSCs (Table I). L-PRP may modulate STAT3 and p27 expression to upregulate the expression of cyclins and cyclin-dependent kinases to increase the proliferation of tendon cells (37). Furthermore, according to Zhou et al (35), L-PRP treatment induced significantly higher proliferation and differentiation of tenocytes compared with P-PRP in a cell culture experiment. However, Zhang et al (28) reported opposite results, namely that L-PRP exerts a harmful effect on TSCs, inhibiting their proliferation, accelerating non-tenocyte differentiation and inducing their apoptosis. Of note, TSCs in that study were isolated from young healthy rabbits, which are different from the cells of older human adults with chronic tendinopathy. A stricter experimental design may be required to obtain evidence on this in the future and research should particularly focus on whether the TSCs were derived from healthy or injured tendons, and whether tendon injury was acute or chronic.

Effect on metabolism. L-PRP has been indicated to affect the metabolism of tendon cells involved in the wound healing 
Table I. Summary of the functions of growth factors (6-9).

Growth

factor

Function

\begin{tabular}{|c|c|}
\hline \multirow[t]{3}{*}{ VEGF } & - Angiogenesis \\
\hline & - Migration and mitosis of endothelial cells \\
\hline & - Chemotactic for macrophages and granulocytes \\
\hline \multirow[t]{3}{*}{ TGF- $\beta$} & - Promotes cellular proliferation and differentiation \\
\hline & Angiogenesis \\
\hline & - Stimulates matrix and collagen synthesis \\
\hline \multirow[t]{2}{*}{ IGF-1 } & $\begin{array}{l}\text { - Promotes cellular growth, proliferation and } \\
\text { differentiation }\end{array}$ \\
\hline & - Stimulates matrix production \\
\hline \multirow[t]{4}{*}{ PDGF } & - Angiogenesis \\
\hline & $\begin{array}{l}\text { - Triggers the activities of neutrophils, fibroblasts } \\
\text { and macrophages }\end{array}$ \\
\hline & - Chemoattractant/cell proliferator \\
\hline & - Regulates the expression of other growth factors \\
\hline \multirow[t]{3}{*}{ bFGF } & - Angiogenesis \\
\hline & - Promotes cellular proliferation and migration \\
\hline & - Promotes collagen production and tissue repair \\
\hline
\end{tabular}

VEGF, endothelial growth factor; TGF- $\beta$, transforming growth factor beta; IGF-1, insulin-like growth factor-1; PDGF, platelet-derived growth factor; bFGF, basic fibroblast growth factor.

process in in vitro and animal studies $(20,23,30,35,38)$. In the proliferation phase, tendon fibroblasts promote the synthesis of abundant type III collagen, granulation tissue and other extracellular matrix (ECM) components (such as proteoglycans) and deposit them to the wound site. In fact, normal tendon consists of type I and type III collagen, with type I collagen being the major component that is responsible for tensile strength (1). When tendons are injured, type I collagen is downregulated and type III collagen synthesis is intensified, and its synthesis occurs earlier in the progress of tendon healing compared with that of type I collagen (39). PRP is known to enhance type I collagen synthesis instead of type III collagen in order to accelerate tendon healing (40). However, leukocyte inclusion in L-PRP has been controversial, as certain studies indicated a beneficial effect and others reported a deleterious effect on tissue regeneration, as leukocytes may cause more inflammation (28).

Certain studies have suggested that L-PRP exerts both catabolic and anabolic effects $(19,41)$, whereas P-PRP exerts mainly anabolic effects on injured tissues. Regarding the anabolic effects of L-PRP, the collagen fiber arrangement time is shortened, which is caused by the direct stimulation of local stem cells and ECM genes to accelerate collagen synthesis via the profibrotic growth factors, such as TGF- $\beta$ and bFGF contained in the L-PRP $(20,22,26)$. Among these, TGF- $\beta$ has been indicated to act in almost all phases of tendon healing and serves as a stimulator of extrinsic cell migration, regulation of proteinases and collagen production (7).

With regard to the catabolic effects, the increased numbers of leukocytes in L-PRP may stimulate fibroblasts to release catabolic cytokines, such as interleukin-1 $\beta$ (IL-1 $\beta$ ), tumor necrosis factor- $\alpha$ (TNF- $\alpha$ ) and matrix metalloproteinases (MMPs), which are capable of degrading the ECM of various tissue types $(26,30,35)$. Regarding catabolic factors, the levels of MMP-1, MMP-3 and MMP-9 were indicated to be strongly correlated with the leukocyte concentration $(26,31,35)$ and they may accelerate the proteolysis of the ECM through invading blood vessel endothelial cells, resulting in impaired mechanical stability. Based on the catabolic effect of leukocytes in PRP, it may be inferred that an increased platelet concentration in L-PRP would have a positive effect by suppressing the expression of catabolic cytokines; however, it was reported that increasing the platelet/leukocyte concentration ratio in L-PRP was not beneficial (42). In addition, based on the current knowledge, it is difficult to determine whether increased gene expression levels of MMP1 and MMP3 may be beneficial for the healing of degenerative or ruptured tendons (26).

Catabolism and anabolism are balanced in normal tendons; however, this balance is disrupted in injured tendons. Whether catabolism or anabolism prevails depends on the different types of tendon injury and healing stage $(23,30)$. In a chronic rabbit Achilles tendinopathy model at 4 weeks after collagenase induction, Yan et al (30) observed that both P-PRP and L-PRP promoted the formation of larger collagen fibrils, but P-PRP exerted a stronger effect than L-PRP. However, in the early model at 1 week after collagenase induction, Li et al (23) detected higher collagen I and lower collagen III content following L-PRP injection compared with that following P-PRP injection.

In fact, the current opinion is that the effect of L-PRP on metabolism is closely associated with the timing and the phase of tendon healing. Application of L-PRP at the early stage of tendon disorders or acute injury causes higher expression of collagen I, while its delivery at a later stage results in higher expression of collagen III $(20,23,30,38)$. In other words, early delivery of L-PRP promotes matrix maturation, while late delivery impairs the matrix modeling process in tendon repair.

Inflammatory response. Tissue healing is a process of inflammation requiring leukocytes, which are contained in L-PRP. In the inflammatory phase, inflammatory cells, including neutrophils, monocytes and lymphocytes, migrate from surrounding tissues to the wound site. Tendon healing begins with local hemostasis, followed by the migration of neutrophils and phagocytes to the lesion site to clear foreign bodies and necrotic tissues (43). Tissue macrophages are known to become activated and polarized into M1 and M2 phenotypes under the influence of the surrounding matrix and environmental factors; in this paradigm, M1 macrophages predominate early and have a proinflammatory function via the release of IL-1 $\beta$, TNF- $\alpha$ and IL-6, while M2 macrophages accumulate later and serve an anti-inflammatory role via the release of IL-10 and TGF- $\beta 1$ (44). Macrophages have key roles in promoting inflammation at the early stage and resolving inflammation at the late stage of tendon healing.

The inclusion of leukocytes in L-PRP remain controversial (14). Regarding the positive effects, inflammation causes release of growth factors and cytokines, which induce neovascularization and chemotaxis of fibroblasts and stimulate collagen synthesis. Furthermore, leukocytes in PRP recruit 


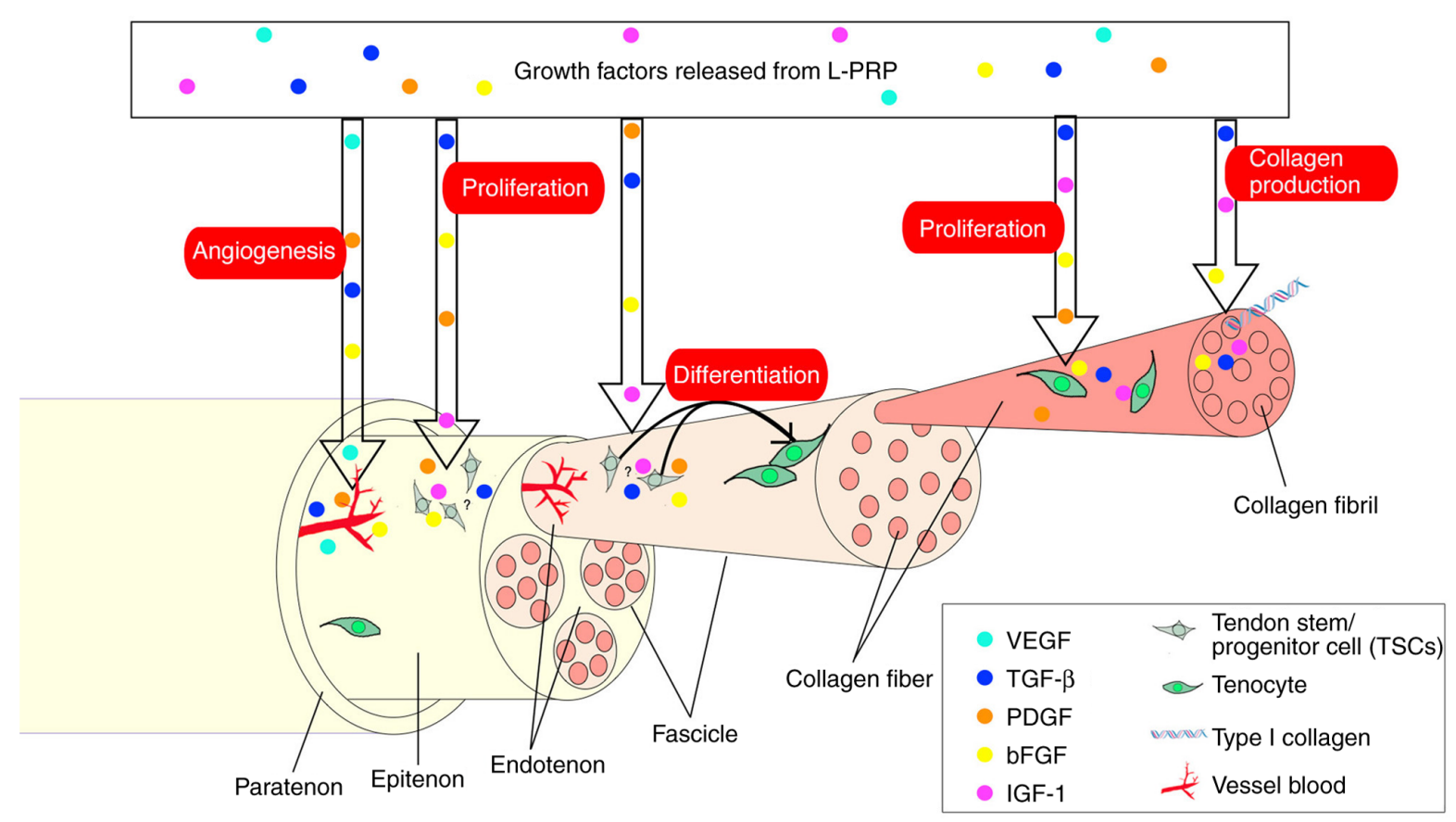

Figure 1. Key molecular, cellular and collagen changes in tendon caused by growth factors released from L-PRP at the early stage. L-PRP promotes angiogenesis, tendon cell proliferation (both TSCs and tenocytes) and differentiation of TSCs into tenocytes, as well as type I collagen production. The exact location of TSCs is still debated (therefore indicated with '?'). L-PRP, leukocyte- and platelet-rich plasma; VEGF, vascular endothelial growth factor; PDGF, platelet-derived growth factor; TSCs, tendon stem/progenitor cells; TGF- $\beta$, transforming growth factor beta; IGF-1, insulin-like growth factor-1; bFGF, basic fibroblast growth factor.

more macrophages through angiogenesis during the early phase of tendon repair (38). Although leukocytes have key roles in tissue repair and provide desirable protection against infectious agents, their proinflammatory and immunological effects may also induce undesirable local cell and tissue damage that compromises the intended healing effect. With regard to the negative effects, leukocytes are considered to be the major source of pro-inflammatory cytokines (such as IL-1 $\beta$, TNF- $\alpha$ and IL-6) and catabolic enzymes, which may cause injury of therapeutic tissue (45).

However, it is well known that controlled inflammation has certain advantages in tissue repair, while excessive or persistent inflammation may be harmful (46). Therefore, whether inflammation is beneficial for tendon healing depends on the different stages of tendon disorders. A previous study demonstrated that delivery of L-PRP at the early rather than the late stage promoted the repair of Achilles tendinopathy in rabbits and suggested that L-PRP may alleviate inflammation at the early stage, whereas L-PRP exerted a less prominent beneficial effect at the late stage of tendinopathy (23). This is consistent with the findings of Zhang et al (28), Zhou et al (35) and Jiang et al (38).

For the treatment of acute tendon injuries, leukocytes in L-PRP may be helpful when inflammation is at a critical stage by inducing a catabolic response, which may clear foreign bodies and necrotic tissues by leukocyte recruitment to the lesion site and by fighting off infectious agents. However, the prolonged duration of infiltration by excessive numbers of neutrophils may impair the healing process as chronic disease replaces acute inflammation. Administration of L-PRP at the later stage would lead to local inflammatory edema after tendon injury, with scar tissue formation and angiofibroblastic dysfunction in chronic tendinopathy homeostasis (30).

\section{Effects of L-PRP on tendon healing based on clinical studies}

General. The clinical manifestations of tendon disease are characterized by pain and dysfunction. The symptoms are well defined and frequently long-lasting, limiting the patients' functioning regarding physical exercise and daily activities.

Pain. L-PRP has been indicated to decrease the pain of patients in the clinical setting $(25,36,47-51)$. According to the classic model, inflammation is responsible for the pain associated with tendon disorders. However, chronically painful tendons may exhibit no evidence of inflammation and there is no associated pain in several intratendinous lesions detected on magnetic resonance imaging (MRI) or ultrasound. It has been proposed that pain may be caused by a combination of mechanical and biochemical factors (52). Tendon degeneration with mechanical breakdown of collagen may theoretically cause pain, whereas chemical irritants and neurotransmitters may also generate pain in tendon disorders.

In one of the largest studies, Mishra et al (49) evaluated 230 patients with lateral epicondylitis who failed to respond to conservative treatment for at least 3 months. Patients treated with L-PRP exhibited a significant improvement in pain compared with the bupivacaine control at 24 weeks. More importantly, in a recent study (36), Pearson's correlation analysis was performed between the clinical score and the biological components and the Pearson correlation coefficient was calculated between the Visual Analogue Scale score and the 


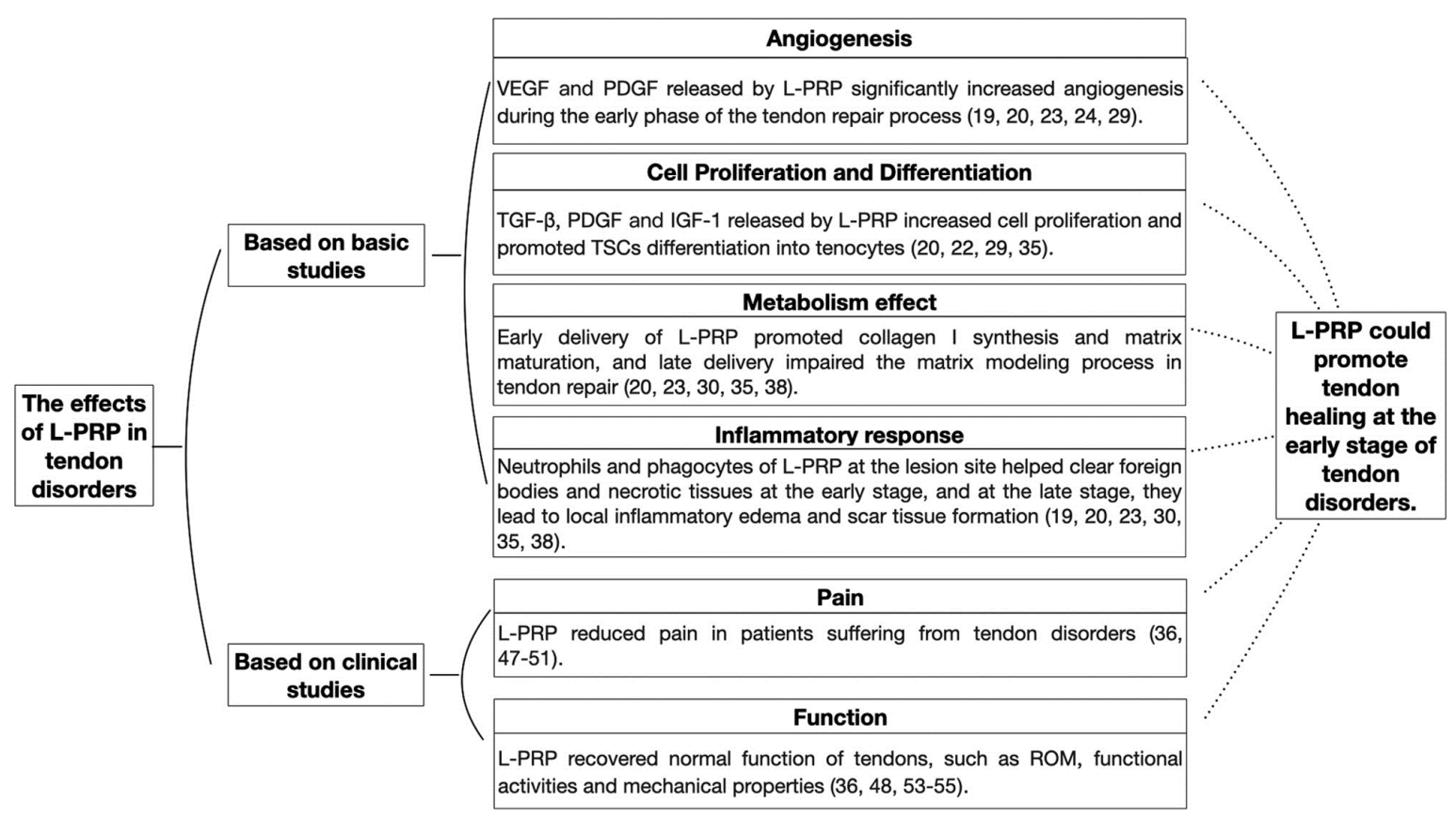

Figure 2. Major effects of L-PRP in tendon disorders according to basic and clinical studies. L-PRP, leukocyte- and platelet-rich plasma; VEGF, vascular endothelial growth factor; PDGF, platelet-derived growth factor; TSCs, tendon stem/progenitor cells; TGF- $\beta$, transforming growth factor beta; IGF-1, insulin-like growth factor-1; ROM, range of joint movement.

leukocyte concentration in L-PRP, demonstrating that L-PRP was highly associated with pain relief in patients suffering from tendon disorders. Of note, in the aforementioned studies, it was observed that pain relief was first observed after 8 weeks after L-PRP was applied to patients with chronic tendon injury for $>3$ months, while pain relief was first observed earlier (seven days later) in patients with acute tendon injury treated with L-PRP $(25,36,47-51)$. However, more research is required to explain this phenomenon.

Function. Certain studies have confirmed that L-PRP may improve tendon function in terms of the range of joint movement (ROM) and the ability to perform physical activities (36,48,53-55). Lim et al (36) indicated that L-PRP enhanced the Modified Mayo Clinic performance scores and MRI grade in patients suffering from lateral epicondylitis for $>3$ months. The study also used Pearson's correlation coefficient to demonstrate the association between the scores and the growth factor levels in PRP, indicating that the TGF- $\beta$ level in L-PRP was highly correlated with the Modified Mayo Clinic performance scores and MRI grade improvement, whereas the VEGF level in L-PRP was highly correlated with MRI grade improvement. Fitzpatrick et al (53) reported that L-PRP improved the modified Harris Hip Scores in patients suffering from gluteus medius and minimus tendinopathy for $>4$ months. The constant score and Disabilities of the Arm, Shoulder and Hand score were tested in patients with rotator cuff tears/lateral epicondylitis who were treated with L-PRP, with increasing scores reflecting less pain, as well as improved ROM and ability to perform physical activities $(48,54)$. Charousset et al (55) also reported that, although there was no significant difference between the L-PRP group and the blank group, the L-PRP group had smaller iterative tears. Collectively, these results indicated that L-PRP may promote the recovery of normal function following tendon injury, as well as the restoration of mechanical properties.

\section{Conclusion and perspectives}

Tendon disorders are a frequently encountered clinical problem that commonly affects athletes and middle-aged patients who do not exercise much, accounting for $30-50 \%$ of all sports-related injuries (56). With the current widespread research indicating that PRP contains a large number of cytokines and growth factors required for tendon healing, PRP therapy has gradually gained popularity in the clinical field (57).

The main effects of L-PRP in tendon disorders according to basic and clinical studies are summarized in Fig. 2. These clinical studies have demonstrated that L-PRP is not only highly associated with pain relief in patients suffering from tendon disorders, but may also help the injured tendon to recover its normal functions, including the ROM, functional activities and mechanical properties (36,47-51,53-55).

Based on basic studies, the underlying mechanisms for the effects of L-PRP likely involve promoting angiogenesis, cell proliferation and differentiation during the process of tendon healing. Growth factors, such as VEGF and PDGF, released from L-PRP, significantly increase angiogenesis during the early phase of the tendon repair process $(19,20,23,24,29)$. Tenocyte proliferation and differentiation of TSCs into tenocytes are crucial for tendon healing and a number of studies have indicated that TGF- $\beta$, PDGF and IGF released by L-PRP accelerate the process $(20,22,29,35)$. Furthermore, regarding metabolism and the inflammatory response, early delivery of L-PRP induced additional inflammation and further release of growth factors, which help clear foreign bodies and necrotic tissues by neutrophils and phagocytes at the lesion site at 
the early stage and accelerate the synthesis of the ECM by transitioning from type III collagen fibers to type I collagen fibers $(19,20,23,30,35,38)$. In addition, L-PRP may enhance antimicrobial functions and increase the concentration of growth factors in platelet concentrate (58).

From the current available literatures, leukocytes in L-PRP are likely beneficial, but their effects depend on the biological state of the injured tissue and its surrounding microenvironment (38). The timing of the L-PRP injection also appears to be important. The conclusion of the present review is that L-PRP exerts beneficial effects by promoting tendon healing at the early stage, whereas it is likely to be detrimental to the tendon at a later stage due to the risk of inducing excessive catabolic and inflammatory responses. Of note, in the studies included, the effect of L-PRP depended on the preparation method, the kit used to prepare L-PRP and the content of leukocytes, as determined by cytology.

In the future, more basic and clinical research should be performed to verify the effect of L-PRP and to clearly determine the conditions under which L-PRP is beneficial for tendon injury, with factors to be considered being the tendon disorder type, stage of tendon healing and inflammation status. In addition, the composition and contents of PRP preparations should be accurately measured in future studies, as the majority of previous clinical studies prepared PRP using different commercial kits and the final product varies in terms of platelet and leukocyte content. Furthermore, the immunomodulatory and metabolic effects of all subpopulations of leukocytes included in the preparations should be explored. Such efforts may help determine the optimal composition of PRP preparations and improve the efficacy of PRP in the treatment of tendon disorders.

\section{Acknowledgements}

Not applicable.

\section{Funding}

No funding was received.

\section{Availability of data and materials}

Not applicable.

\section{Authors' contributions}

XLL, RZ and XYL designed the present article. XLL and RZ drawn the manuscript. BZ, YJL, SG and CLW a literature search and selected the studies to be performed XLL, DXW and SL revised including the manuscript. Data authentication is not applicable. All authors approved the final version of the article.

\section{Ethics approval and consent to participate}

Not applicable.

\section{Patient consent for publication}

Not applicable.

\section{Competing interests}

The authors declare that they have no competing interests.

\section{References}

1. Wang JH: Mechanobiology of tendon. J Biomech 39: 1563-1582, 2006.

2. Clayton RA and Court-Brown CM: The epidemiology of musculoskeletal tendinous and ligamentous injuries. Injury 39: 1338-1344, 2008.

3. Nourissat G, Berenbaum F and Duprez D: Tendon injury: From biology to tendon repair. Nat Rev Rheumatol 11: 223-233, 2015.

4. Costa-Almeida R, Babo PS, Reis RL and Gomes ME: Platelet-rich blood derivatives for tendon regeneration. J Am Acad Orthop Surg 28: e202-e205, 2020.

5. Zhou Y and Wang JH: PRP treatment efficacy for tendinopathy: A review of basic science studies. Biomed Res Int 2016: 9103792 , 2016.

6. Eppley BL, Woodell JE and Higgins J: Platelet quantification and grow th factor analysis from platelet-rich plasma: Implications for wound healing. Plast Reconstr Surg 114: 1502-1508, 2004.

7. Molloy T, Wang Y and Murrell G: The roles of growth factors in tendon and ligament healing. Sports Med 33: 381-394, 2003.

8. Wu PI, Diaz R and Borg-Stein J: Platelet-rich plasma. Phys Med Rehabil Clin N Am 27: 825-853, 2016.

9. Jeong DU, Lee CR, Lee JH, Pak J, Kang LW, Jeong BC and Lee SH: Clinical applications of platelet-rich plasma in patellar tendinopathy. Biomed Res Int 2014: 249498, 2014.

10. Dohan Ehrenfest DM, Rasmusson L and Albrektsson T: Classification of platelet concentrates: From pure platelet-rich plasma (P-PRP) to leucocyte- and platelet-rich fibrin (L-PRF). Trends Biotechnol 27: 158-167, 2009.

11. Krogh TP, Fredberg U, Stengaard-Pedersen K, Christensen R, Jensen $P$ and Ellingsen T: Treatment of lateral epicondylitis with platelet-rich plasma, glucocorticoid, or saline: A randomized, double-blind, placebo-controlled trial. Am J Sports Med 41: 625-635, 2013 .

12. de Vos RJ, Weir A, van Schie HT, Bierma-Zeinstra SM, Verhaar JA, Weinans H and Tol JL: Platelet-rich plasma injection for chronic Achilles tendinopathy: A randomized controlled trial. JAMA 303: 144-149, 2010.

13. de Jonge S, de Vos RJ, Weir A, van Schie HT, Bierma-Zeinstra SM, Verhaar JA, Weinans H and Tol JL: One-year follow-up of platelet-rich plasma treatment in chronic Achilles tendinopathy: A double-blind randomized placebo-controlled trial. Am J Sports Med 39: 1623-1629, 2011.

14. Castillo TN, Pouliot MA, Kim HJ and Dragoo JL: Comparison of growth factor and platelet concentration from commercial platelet-rich plasma separation systems. Am J Sports Med 39: 266-271, 2011.

15. Baksh N, Hannon CP, Murawski CD, Smyth NA and Kennedy JG: Platelet-rich plasma in tendon models: A systematic review of basic science literature. Arthroscopy 29: 596-607, 2013.

16. Chen X, Jones IA, Park C and Vangsness CT Jr: The efficacy of platelet-rich plasma on tendon and ligament healing: A systematic review and meta-analysis with bias assessment. Am J Sports Med 46: 2020-2032, 2018.

17. Fitzpatrick J, Bulsara $\mathrm{M}$ and Zheng MH: The Effectiveness of Platelet-rich plasma in the treatment of tendinopathy: A Meta-analysis of randomized controlled clinical trials. Am J Sports Med 45: 226-233, 2017.

18. Hope M and Saxby TS: Tendon healing. Foot Ankle Clin 12: 553-567, 2007.

19. Dragoo JL, Braun HJ, Durham JL, Ridley BA, Odegaard JI, Luong R and Arnoczky SP: Comparison of the acute inflammatory response of two commercial platelet-rich plasma systems in healthy rabbit tendons. Am J Sports Med 40: 1274-1281, 2012.

20. Kobayashi Y, Saita Y, Takaku T, Yokomizo T, Nishio H, Ikeda H, Takazawa Y, Nagao M, Kaneko K and Komatsu N: Platelet-rich plasma (PRP) accelerates murine patellar tendon healing through enhancement of angiogenesis and collagen synthesis. J Exp Orthop 7: 49, 2020.

21. Lyras DN, Kazakos K, Agrogiannis G, Verettas D, Kokka A, Kiziridis G, Chronopoulos E and Tryfonidis M: Experimental study of tendon healing early phase: Is IGF-1 expression influenced by platelet rich plasma gel? Orthop Traumatol Surg Res 96: 381-387, 2010. 
22. Lyras DN, Kazakos K, Tryfonidis M, Agrogiannis G, Botaitis S, Kokka A, Drosos G, Tilkeridis K and Verettas D: Temporal and spatial expression of TGF-betal in an Achilles tendon section model after application of platelet-rich plasma. Foot Ankle Surg 16: 137-141, 2010.

23. Li S, Wu Y, Jiang G, Tian X, Hong J, Chen S, Yan R, Feng G and Cheng Z: Intratendon delivery of leukocyte-rich platelet-rich plasma at early stage promotes tendon repair in a rabbit Achilles tendinopathy model. J Tissue Eng Regen Med 14: 452-463, 2020.

24. Nishio H, Saita Y, Kobayashi Y, Takaku T, Fukusato S, Uchino S Wakayama T, Ikeda $\mathrm{H}$ and Kaneko K: Platelet-rich plasma promotes recruitment of macrophages in the process of tendon healing. Regen Ther 14: 262-270, 2020.

25. Sharma $P$ and Maffulli N: Tendon injury and tendinopathy: Healing and repair. J Bone Joint Surg Am 87: 187-202, 2005

26. de Mos M, van der Windt AE, Jahr H, van Schie HT, Weinans $H$, Verhaar JA and van Osch GJ: Can platelet-rich plasma enhance tendon repair? A cell culture study. Am J Sports Med 36 : $1171-1178,2008$

27. Mazzocca AD, McCarthy MB, Chowaniec DM, Dugdale EM, Hansen D, Cote MP, Bradley JP, Romeo AA, Arciero RA and Beitzel K: The positive effects of different platelet-rich plasma methods on human muscle, bone, and tendon cells. Am J Sports Med 40: 1742-1749, 2012

28. Zhang L, Chen S, Chang P, Bao N, Yang C, Ti Y, Zhou L and Zhao J: Harmful effects of Leukocyte-rich platelet-rich plasma on rabbit tendon stem cells in vitro. Am J Sports Med 44: 1941-1951, 2016.

29. Lyras DN, Kazakos K, Verettas D, Botaitis S, Agrogiannis G, Kokka A, Pitiakoudis M and Kotzakaris A: The effect of platelet-rich plasma gel in the early phase of patellar tendon healing. Arch Orthop Trauma Surg 129: 1577-1582, 2009.

30. Yan R, Gu Y, Ran J, Hu Y, Zheng Z, Zeng M, Heng BC, Chen X, Yin Z, Chen W, et al: Intratendon delivery of leukocyte-poor platelet-rich plasma improves healing compared with leukocyte-rich platelet-rich plasma in a rabbit Achilles tendinopathy model. Am J Sports Med 45: 1909-1920, 2017.

31. Kobayashi Y, Saita Y, Nishio H, Ikeda H, Takazawa Y, Nagao M, Takaku T, Komatsu N and Kaneko K: Leukocyte concentration and composition in platelet-rich plasma (PRP) influences the growth factor and protease concentrations. J Orthop Sci 21: 683-689, 2016

32. Denapoli PM, Stilhano RS, Ingham SJ, Han SW and Abdalla RJ Platelet-rich plasma in a murine model: Leukocytes, growth factors, Flt-1, and muscle healing. Am J Sports Med 44: 1962-1971, 2016

33. Zhang J and Wang JH: Characterization of differential properties of rabbit tendon stem cells and tenocytes. BMC Musculoskelet Disord 11: 10, 2010

34. Zhang $\mathbf{J}$ and Wang $\mathrm{JH}$ : Platelet-rich plasma releasate promotes differentiation of tendon stem cells into active tenocytes. Am J Sports Med 38: 2477-2486, 2010.

35. Zhou Y, Zhang J, Wu H, Hogan MV and Wang JH: The differential effects of leukocyte-containing and pure platelet-rich plasma (PRP) on tendon stem/progenitor cells-implications of PRP application for the clinical treatment of tendon injuries. Stem Cell Res Ther 6: 173, 2015.

36. Lim W, Park SH, Kim B, Kang SW, Lee JW and Moon YL: Relationship of cytokine levels and clinical effect on platelet-rich plasma-treated lateral epicondylitis. J Orthop Res 36: 913-920, 2018

37. Yu TY, Pang JH, Wu KP, Lin LP, Tseng WC and Tsai WC: Platelet-rich plasma increases proliferation of tendon cells by modulating Stat 3 and p27 to up-regulate expression of cyclins and cyclin-dependent kinases. Cell Prolif 48: 413-420, 2015.

38. Jiang G, Wu Y, Meng J, Wu F, Li S, Lin M, Gao X, Hong J, Chen W, Yan S, et al: Comparison of leukocyte-rich platelet-rich plasma and leukocyte-poor platelet-rich plasma on achilles tendinopathy at an early stage in a rabbit model. Am J Sports Med 48: $1189-1199,2020$

39. Gaut L and Duprez D: Tendon development and diseases. Wiley Interdiscip Rev Dev Biol 5: 5-23, 2016.

40. Alsousou J, Thompson M, Harrison P, Willett K and Franklin S Effect of platelet-rich plasma on healing tissues in acute ruptured Achilles tendon: A human immunohistochemistry study. Lancet 385 (Suppl 1): S19, 2015.
41. Filardo G, Kon E, Pereira Ruiz MT, Vaccaro F, Guitaldi R, Di Martino A, Cenacchi A, Fornasari PM and Marcacci M: Platelet-rich plasma intra-articular injections for cartilage degeneration and osteoarthritis: Single-versus double-spinning approach. Knee Surg Sports Traumatol Arthrosc 20: 2082-2091, 2012.

42. McCarrel TM, Minas T and Fortier LA: Optimization of leukocyte concentration in platelet-rich plasma for the treatment of tendinopathy. J Bone Joint Surg Am 94: e143, 2012.

43. Butler DL, Juncosa N and Dressler MR: Functional efficacy of tendon repair processes. Annu Rev Biomed Eng 6: 303-329, 2004.

44. Stolk M, Klatte-Schulz F, Schmock A, Minkwitz S, Wildemann B and Seifert M: New insights into tenocyte-immune cell interplay in an in vitro model of inflammation. Sci Rep 7: 9801, 2017.

45. Sunwoo JY, Eliasberg CD, Carballo CB and Rodeo SA: The role of the macrophage in tendinopathy and tendon healing. J Orthop Res 38: 1666-1675, 2020.

46. Dakin SG, Dudhia J and Smith RK: Resolving an inflammatory concept: The importance of inflammation and resolution in tendinopathy. Vet Immunol Immunopathol 158: 121-127, 2014.

47. D'Ambrosi R, PalumboF, Paronzini A, Ragone V and Facchini RM: Platelet-rich plasma supplementation in arthroscopic repair of full-thickness rotator cuff tears: A randomized clinical trial. Musculoskelet Surg 100: 25-32, 2016.

48. Zhang Z, Wang Y and Sun J: The effect of platelet-rich plasma on arthroscopic double-row rotator cuff repair: A clinical study with 12-month follow-up. Acta Orthop Traumatol Turc 50: 191-197, 2016.

49. Mishra AK, Skrepnik NV, Edwards SG, Jones GL, Sampson S, Vermillion DA, Ramsey ML, Karli DC and Rettig AC: Efficacy of platelet-rich plasma for chronic tennis elbow: A double-blind, prospective, multicenter, randomized controlled trial of 230 patients. Am J Sports Med 42: 463-471, 2014.

50. Mishra A and Pavelko T: Treatment of chronic elbow tendinosis with buffered platelet-rich plasma. Am J Sports Med 34: 1774-1778, 2006

51. Thanasas C,Papadimitriou G,Charalambidis C,Paraskevopoulos I and Papanikolaou A: Platelet-rich plasma versus autologous whole blood for the treatment of chronic lateral elbow epicondylitis: A randomized controlled clinical trial. Am J Sports Med 39: 2130-2134, 2011.

52. Khan KM, Cook JL, Bonar F, Harcourt P and Astrom M Histopathology of common tendinopathies: Update and implications for clinical management. Sports Med 27: 393-408, 1999.

53. Fitzpatrick J, Bulsara MK, O'Donnell J and Zheng MH: Leucocyte-rich platelet-rich plasma treatment of gluteus medius and minimus tendinopathy: A double-blind randomized controlled trial with 2-year follow-up. Am J Sports Med 47: $1130-1137,2019$

54. Gosens T, Peerbooms JC, van Laar W and den Oudsten BL: Ongoing positive effect of platelet-rich plasma versus corticosteroid injection in lateral epicondylitis: A double-blind randomized controlled trial with 2-year follow-up. Am J Sports Med 39: 1200-1208, 2011.

55. Charousset C, Zaoui A, Bellaiche L and Piterman M: Does autologous leukocyte-platelet-rich plasma improve tendon healing in arthroscopic repair of large or massive rotator cuff tears? Arthroscopy 30: 428-435, 2014.

56. Jarvinen TA, Kannus P, Maffulli N and Khan KM: Achilles tendon disorders: Etiology and epidemiology. Foot Ankle Clin 10: 255-266, 2005 .

57. Wasterlain AS, Braun HJ, Harris AH, Kim HJ and Dragoo JL: The systemic effects of platelet-rich plasma injection. Am J Sports Med 41: 186-193, 2013.

58. Moojen DJ, Everts PA, Schure RM, Overdevest EP, van Zundert A, Knape JT, Castelein RM, Creemers LB and Dhert WJ: Antimicrobial activity of platelet-leukocyte gel against Staphylococcus aureus. J Orthop Res 26: 404-410, 2008.

This work is licensed under a Creative Commons Attribution-NonCommercial-NoDerivatives 4.0 International (CC BY-NC-ND 4.0) License. 